\title{
Microscaled and nanoscaled platinum sensors
}

\author{
Aditya Rajagopal, ${ }^{a}$ Sameer Walavalkar, Samson Chen, Luke Guo, Tom Gwinn, and \\ Axel Scherer \\ Electrical Engineering and Applied Physics, Kavli Nanoscience Institute, Caltech Pasadena, \\ California 91125, USA
}

(Received 4 April 2010; accepted 5 September 2010; published online 28 September 2010)

\begin{abstract}
We show small and robust platinum resistive heaters and thermometers that are defined by microlithography on silicon substrates. These devices can be used for a wide range of applications, including thermal sensor arrays, programmable thermal sources, and even incandescent light emitters. To explore the miniaturization of such devices, we have developed microscaled and nanoscaled platinum resistor arrays with wire widths as small as $75 \mathrm{~nm}$, fabricated lithographically to provide highly localized heating and accurate resistance (and hence temperature) measurements. We present some of these potential applications of microfabricated platinum resistors in sensing and spectroscopy. (C) 2010 American Institute of Physics. [doi:10.1063/1.3494088]
\end{abstract}

Microlithography and nanolithography techniques can now be applied toward the miniaturization of a wide variety of sensors and actuators, leading to their integration into chip-based analysis systems. On-chip sensors enable the monitoring and regulation of many chemical and biological samples in parallel, and reduce the individual device cost, following the trend toward more complex and functional microelectronics through lithographic printing. Specifically, platinum wires have been used for resistive heaters and incandescent light sources since the early 1820s. Platinum does not oxidize, making it a good candidate for vacuum-free, miniaturized visible and infrared sources, heaters, and thermometers. Applications of these microscale thermal control systems include chemical analysis, gas chromatography, ${ }^{1-4}$ microcalorimetry as well as thermal regulation of polymerase chain reactors ${ }^{5}$ and even micropropulsion systems. ${ }^{6}$ Miniaturization of these devices is particularly beneficial for systems that require independent thermal control over many reactions or wide band spectroscopic light sources; the low thermal mass of microfabricated heaters enables greater accuracy in measurement, faster heating and cooling rates, while requiring lower power than macroscopic systems.

Platinum resistance thermometer devices (RTDs) have a linear temperature response in the range of $(-200)-500{ }^{\circ} \mathrm{C}$, and are well suited for the thermal measurement and control of wide array of chemical processes. ${ }^{5}$ In particular, platinum RTDs exhibit a high accuracy and repeatability of temperature measurements when compared with thermocouples for temperatures below $600{ }^{\circ} \mathrm{C}^{7}$ In this paper, we present onchip thin-film, micron-sized platinum resistive thermometers as convenient on-chip thermal control systems and IR light sources.

We fabricated arrays of platinum microresistors and nanoresistors on alumina coated oxidized silicon wafer substrates. Fabrication starts with the growth of $160 \mathrm{~nm}$ of wet thermal oxide on a $\langle 100\rangle$ silicon wafer. Subsequently, a $150 \mathrm{~nm}$ layer of alumina $\left(\mathrm{Al}_{2} \mathrm{O}_{3}\right)$ and a $150 \mathrm{~nm}$ layer of platinum were sputter deposited on the surface using a direct current (dc) magnetron sputtering source. ${ }^{8}$ The approximate film thicknesses were confirmed by scanning electron mi-

${ }^{a)}$ Electronic mail: arajagop@caltech.edu. croscopy. After the complete stack of materials was deposited, a milling mask pattern was then defined using standard photo-lithographic techniques. The resistor pattern was transferred by milling through the platinum and aluminum oxide, into the glass (to remove shunt thermal resistances), using a radio frequency (rf) plasma-based argon mill (Fig. 1 top left inset). After the plasma-milling step, the cross-linked resist milling-mask was removed by exposing the chips to a lowvoltage rf oxygen plasma. These platinum resistors consist of a series of twenty serpentine platinum wires with widths ranging from 1-4 $\mu \mathrm{m}$, and cover areas of $100 \times 200 \mu \mathrm{m}^{2}$ on-chip area (Fig. 1). Finally, selected microresistors were then further postprocessed to create nanometer-wide "nanoresistors" (Fig. 2) by using an FEI Nova200 focused ion beam (FIB) system. The resistor linewidths were reduced from 1-4 $\mu \mathrm{m}$ to dimensions as small as $75 \mathrm{~nm}$. This technique was utilized since it allows for mesoscaled structures to be fabricated using the same set of optical lithography masks.

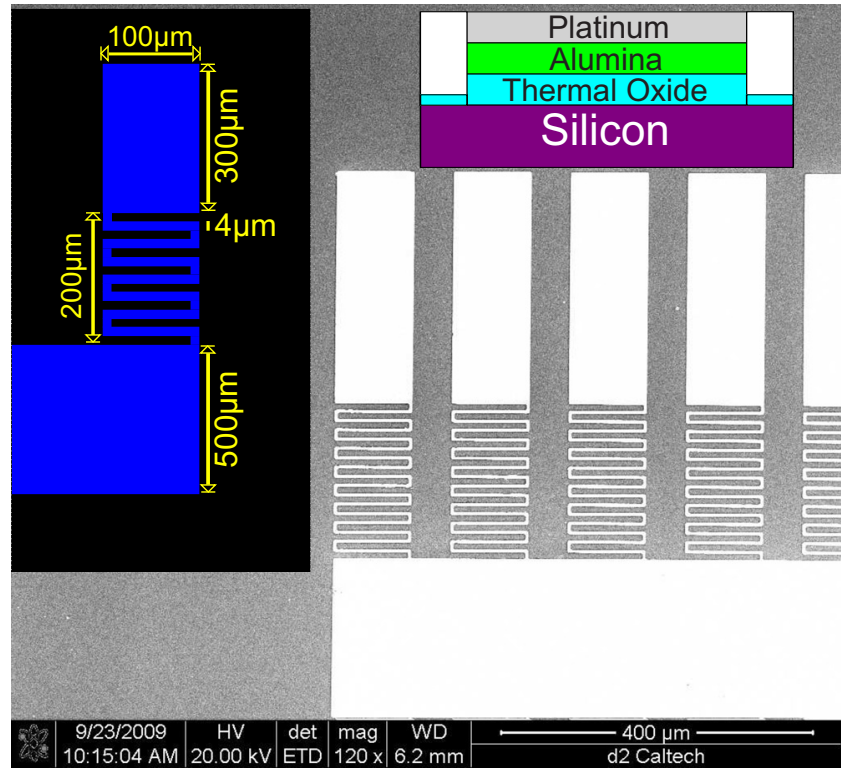

FIG. 1. (Color online) Scanning electron micrograph of platinum microresistors (Inset: top right, resistor element cross section; top left, resistor element dimensions). 


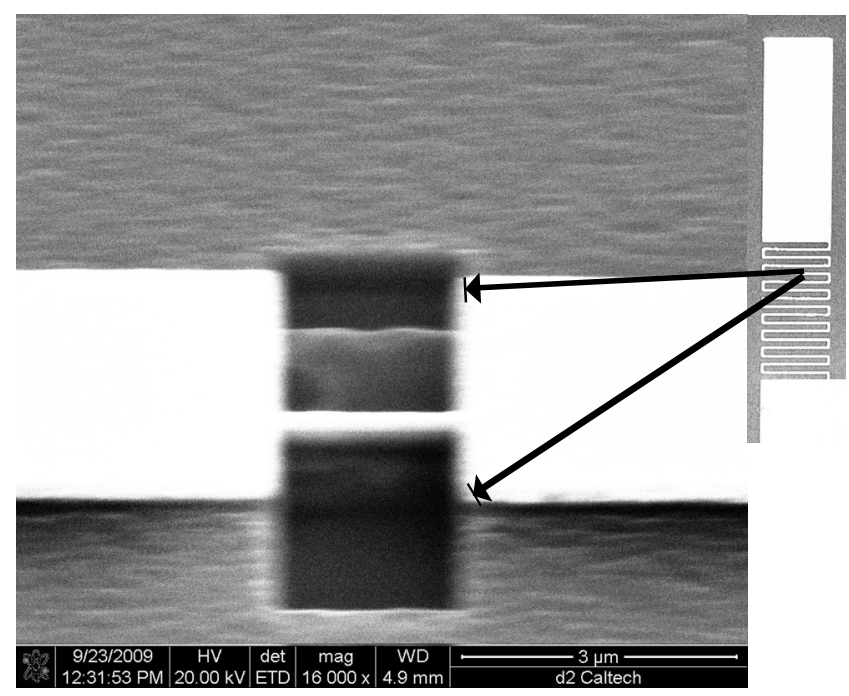

FIG. 2. Scanning electron micrograph of platinum nanobulb (FIB thinned microresistor).

Before temperature-benchmarking, the microresistors were annealed by resistive-heating with a constant power of $\sim 1.5 \mathrm{~W}$ for $10 \mathrm{~min}$ to ensure thermal stability. These annealed resistors show considerably better relative tolerances on each chip than unannealed resistors with a standard deviation of resistance of $\sigma=2.1 \%$ (sample size of $n=20$ ) for annealed resistors and a standard deviation of resistance of $\sigma=48 \%$ (sample size of $\mathrm{n}=194$ ) for unannealed resistors. We believe that this annealing step allows the platinum thin-film to electromigrate in a controlled way and improves the reliability of the resulting heaters. This helps to minimize resistor failures during subsequent heating and measurement and has allowed the annealed resistors to be driven at higher powers than nonannealed resistors.

To test the heating and temperature sensing capabilities of the resistors, a dc power supply was connected to the heater resistor and adjusted to deliver power in steps of $250 \mathrm{~mW}$. The resistance of each adjacent resistor was manually probed and measured in order to characterize the thermal profile of a platinum resistor bank. By taking advantage of the linear temperature coefficient of resistance (TCR) [0.003927 $(\Omega \mathrm{cm}) / \mathrm{C}]$ (Ref. 9) of platinum, we can relate resistance measurements of the platinum structure to the

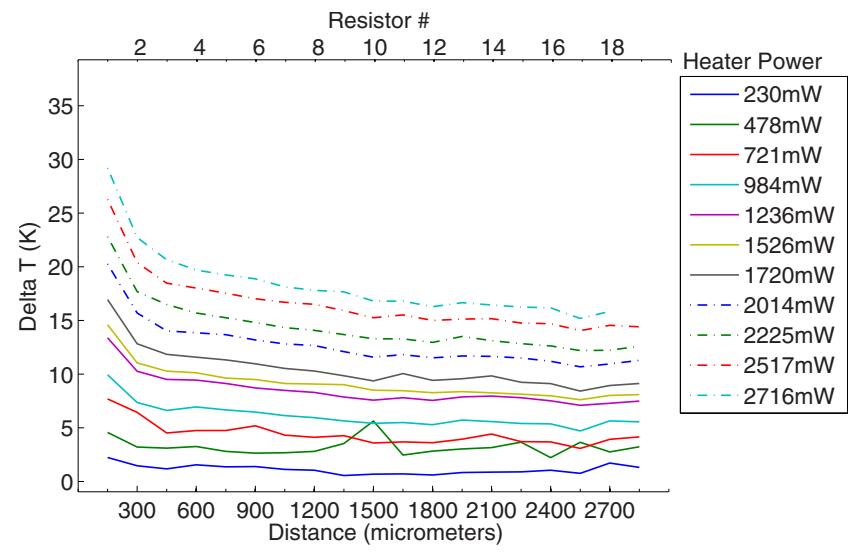

FIG. 3. (Color online) Temperature vs distance for resistor array [R0 is the heating element (located at distance 0), R1-R19 are measurements elements] for various driving powers.

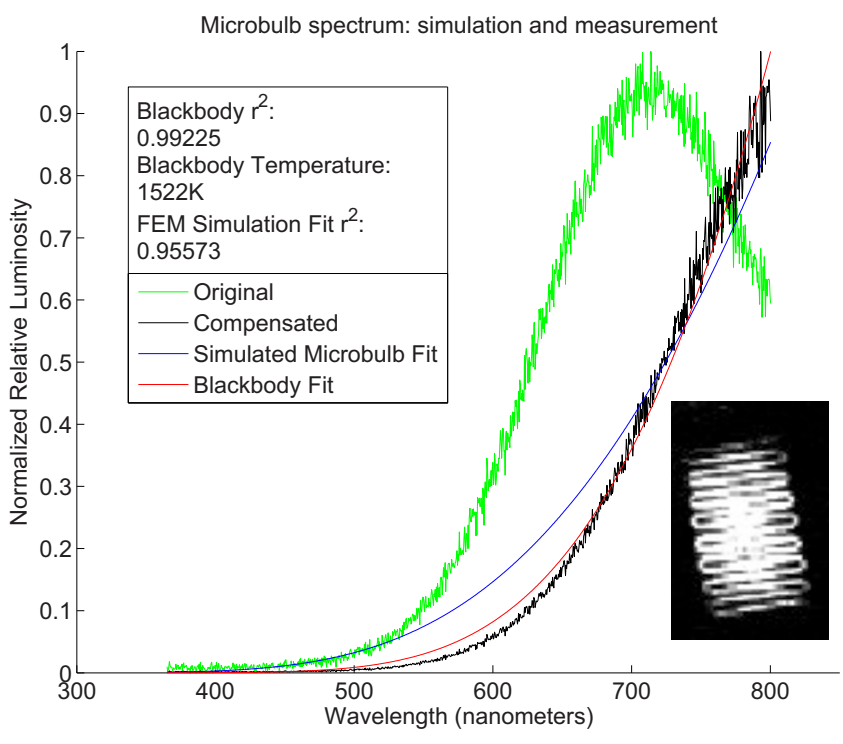

FIG. 4. (Color online) Normalized blackbody emission spectrum of platinum microbulb, and finite element methods extracted spectrum.

temperature of the substrate. The resulting temperature profile across the resistor array (Fig. 3) shows that the temperature decreases with distance from the heating element and with decreasing heater power. Thermal isolation, provided by the $\mathrm{SiO}_{2}$ film, ensures that the heating is confined to the surface $\mathrm{Al}_{2} \mathrm{O}_{3}$.

Furthermore, the platinum resistor structures can be driven with enough power to exhibit luminescence in the visible range (Fig. 4). This process can be enhanced by creating small, highly resistive regions within the platinum conductors. The intense localized heating of the platinum structures caused them to emit as blackbodies with components in the orange-red region of the visible spectrum. Spectra of light emission from these incandescent Pt filaments were extracted using an Acton cooled charge-coupled device camera. We compensated for the spectral sensitivity of the camera and optical system using a $3100 \mathrm{~K}$ near-blackbody light source. Furthermore, we were able to characterize the peak unnormalized (i.e., not corrected for detector optics) emission frequencies as a function of applied filament power across an individual resistor, and these have been summarized in Table I.

Using standard finite element analysis techniques, we were able to validate these spectral measurements. We modeled conduction, radiation, convection, and Joule heating using a nominal $1 \mathrm{~cm} \times 1 \mathrm{~cm}$ chip with microresistors and a $5 \mathrm{~W}$ power source. Our model includes the chip mount used during the measurements, which was modeled as a heat sink

TABLE I. Peak emission wavelength vs power dissipation.

\begin{tabular}{cc}
\hline \hline $\begin{array}{c}\text { Measured peak wavelength } \\
(\mathrm{nm})\end{array}$ & $\begin{array}{c}\text { Input power } \\
(\mathrm{W})\end{array}$ \\
\hline $719.28^{\mathrm{a}}$ & 5.40 \\
$718.02^{\mathrm{a}}$ & 5.92 \\
$706.67^{\mathrm{a}}$ & 6.62 \\
$701.62^{\mathrm{a}}$ & 7.22 \\
$733.36^{\mathrm{b}}$ & 38.57 \\
\hline \hline
\end{tabular}

\footnotetext{
${ }^{\mathrm{a} D e n o t e s ~ n a n o b u l b . ~}$
}

${ }^{b}$ Denotes macrobulb. 
with $9000 \mathrm{~W} / \mathrm{K} \mathrm{m}^{2}$ (as measured). The simulated blackbody emission spectrum, produced using the surface temperatures created by the chip is shown in Fig. 4. The simulated peak temperature was approximately $1900 \mathrm{~K}$, slightly above the melting point of platinum. After optical compensation for our measured spectra, we found that the simulated peak, and the predicted peak from Wein's law matched well with our measurements (see Fig. 4). ${ }^{10,11}$ From this data, we conclude that the platinum filaments are heated slightly beyond their melting temperature, causing them to radiate in the near-visible and visible frequencies.

These high temperatures eventually led the bulb to failure, most likely caused by the melting and evaporation of the platinum from the substrate material. In the future, active cooling or physical confinement of the platinum through encapsulation might be employed to mitigate this failure.

The on-silicon fabrication of the resistive elements allows for integration of complex control circuitry for thermal control. For example, we envision that such a device can be used for applications such as microcalorimetry. These resistor arrays can be used to quantify the exothermic or endothermic nature of reactions. Ultimately, when electronics and fluidics are integrated with these platinum heaters, we envision these platinum resistance thermometers within chipbased gas chromatography systems, accurate thermal controllers for microscopic polymerase chain reactors, ${ }^{12-14}$ and black-body emitter light sources for visible and mid-IR spectroscopy. ${ }^{15-18}$

The authors would like to acknowledge Michael David Henry, Bophan Chhim, Melissa Melendes, and the Kavli Nanoscience Institute for help with fabrication of the devices. Furthermore, the authors want to acknowledge Professor Joseph Shepherd and Philipp Boettcher for the use of an IR pyrometer that was used to calibrate temperature mea- surements. Furthermore, we would like to thank Claudia Shin, Raymond Jimenez, Teresa Emery, and Greg Lutrell for helpful insights. This work was supported by a grant from the Boeing Corporation for the investigations into miniaturized sensor systems for mobile platforms (BOEING CT-BA-GTA-1).

${ }^{1}$ S. Semancik and R. Cavicchi, Acc. Chem. Res. 31, 279 (1998).

${ }^{2}$ I. Simon, N. Barson, M. Bauer, and U. Weimar, Sens. Actuators B 73, 1 (2001)

${ }^{3}$ P. M. Sarro, A. W. van Herwaarden, and W. van der Vlist, Sens. Actuators, A 42, 666 (1994).

${ }^{4}$ J. Yeom, C. R. Field, B. Bae, R. I. Masel, and M. A. Shannon, J. Micromech. Microeng. 18, 125001 (2008).

${ }^{5}$ C. Zhang and D. Xing, Nucleic Acids Res. 35, 4223 (2007).

${ }^{6}$ C. Rossi, D. Briand, M. Dumonteuil, T. Camps, P. Q. Pham, and N. F. de Rooij, Sens. Actuators, A 126, 241 (2006).

${ }^{7}$ J. Courbat, D. Briand, and N. F. de Rooij, Sens. Actuators, A 142, 284 (2008).

${ }^{8}$ M. D. Henry, S. Walavalkar, A. Homyk, and A. Scherer, Nanotechnology 20, 255305 (2009).

${ }^{9}$ CRC Handbook of Chemistry and Physics, edited by R. Weast (CRC, Boca Raton, 1983), p. F-125.

${ }^{10}$ CRC Handbook of Chemistry and Physics, edited by R. Weast (CRC, Boca Raton, 2001), p. F-110.

${ }^{11}$ C. H. Mastrangelo and R. S. Muller, Proceedings of the International Electron Devices Meeting, 1989.

${ }^{12}$ P. Furjes, C. Dusco, M. Adam, J. Zeettner, and I. Barsony, Superlattices Microstruct. 35, 455 (2004).

${ }^{13}$ J. El-Ali, I. R. Perch-Nielsen, C. R. Poulsen, D. D. Bang, P. Telleman, and A. Wolff, Sens. Actuators, A 110, 3 (2004).

${ }^{14}$ D. Briand, S. Heimgartner, M. A. Gretillat, B. van der Schoot, and N. F. de Rooij, J. Micromech. Microeng. 12, 971 (2002).

${ }^{15}$ S. H. Kong, D. D. L. Wijngaards, and R. F. Wolffenbuttel, Sens. Actuators, A 92, 88 (2001).

${ }^{16}$ A. Nitkowski and M. Lipson, Proceedings of the Conference on Lasers and Electro-Optics, 2008.

${ }^{17}$ B. Fischer, A. Behrends, D. Freund, D. F. Lupton, and J. Merker, Platinum Met. Rev. 43, 18 (1999).

${ }^{18}$ W. E. Forsythe and E. Q. Adams, J. Opt. Soc. Am. 35, 108 (1945). 